\section{LfDI NRW: 21. Tätigkeitsbericht 2011/2012}

Am 07.05.2013 legte der Landesbeauftragte für Datenschutz- und Informationsfreiheit Nordrhein-Westfalen, Ulrich Lepper, seinen 21. Tätigkeitsbericht vor. Sein Credo: Die richtigen Maßstäbe finden: Chancen für Datenschutz nutzen, Aktionismus vermeiden, Transparenz schaffen.

Das sind die Schwerpunkte des Berichtes:

Einheitlicher Datenschutz in Europa auf hohem Niveau als Chance: Wirtschaftsinteressen dürfen nicht im Vordergrund stehen.

Viele interessierte Stellen begleiten die Datenschutzgrundverordnung, die den Datenschutz in der ganzen EU einheitlich regeln soll. Einige Unternehmen, unter anderem mit Firmensitz in den USA, haben dabei ein Interesse an einem niedrigen Datenschutzniveau.

Lepper: „Angesichts der Vielfalt der Änderungsvorschläge darf der rote Faden nicht aus den Augen verloren werden: Es kommt auf einen angemessenen Ausgleich zwischen den berechtigten Wirtschaftsinteressen und der informationellen Selbstbestimmung an. Es geht um einen praktikablen Datenschutz auf hohem Niveau und nicht um das Bedienen von Lobbyinteressen. Ich habe Vorschläge zur Verbesserung der Verordnung gemacht."

Videoüberwachung: kein Allheilmittel - Besonnenheit statt Aktionismus

Bei jeder Gelegenheit - zuletzt nach dem Anschlag in Boston - wird mehr Videoüberwachung im öffentlichen Straßenraum gefordert. „Dabei ist mittlerweile nachgewiesen, dass ein Mehr an Videoüberwachung nicht zu einem Weniger an Kriminalität führt; so einfach ist die Welt leider nicht", führt Ulrich Lepper aus.

Auch Arbeitgeber haben wohl nach wie vor eine Vorliebe für das Instrument der Videoüberwachung. „Das Ausforschen von Beschäftigten ist nicht hinnehmbar", so Lepper.

Open Data: mehr Transparenz bei behördlichen Datenbeständen

Positive Ansätze aus Sicht der Informationsfreiheit stellt Ulrich Lepper beim Thema „Open Data“ und der entsprechenden Landtagsinitiative fest: „Das Informationsfreiheitsgesetz des Landes sollte im Sinne einer Veröffentlichungspflicht weiterentwickelt werden. Viele Daten der Verwaltung würden dann transparent, ohne dass dazu ein besonderer Antrag gestellt werden müsste."

Der Landesbeauftragte berichtet dem Landtag und der Öffentlichkeit alle zwei Jahre über seine Tätigkeit. Der Bericht kann bestellt oder heruntergeladen werden unter www.ldi.nrw.de.

\section{LfD Baden-Württemberg: Gesetzgeber zur Regulierung sozialer Netzwerke gefordert}

Der Landesbeauftragte für den Datenschutz, Jörg Klingbeil, hat am 08.05.2013 das Scheitern der freiwilligen Selbstregulierung sozialer
Netzwerke, über die seit November 2011 unter Federführung der Freiwilligen Selbstkontrolle Multimedia-Diensteanbieter (FSM) verhandelt worden war, bedauert und zugleich die Untätigkeit des Gesetzgebers kritisiert: „Die großen internationalen Online-Netzwerke haben die deutsche Politik, vor allem den Bundesinnenminister, erbarmungslos vorgeführt. Die von vornherein bestehenden Befürchtungen der Datenschutzbeauftragten, dass die globalen Player wie Facebook und Google nur auf Zeit spielen und sich nicht einbinden lassen wollen, haben sich leider bewahrheitet. Nun ist umso mehr der Gesetzgeber gefordert, um endlich nutzerfreundliche Regeln durchzusetzen." Jörg Klingbeil erinnerte daran, dass seit fast zwei Jahren ein Gesetzentwurf des Bundesrats zur Änderung des Telemediengesetzes (BR-Drucksache 156/11') auf Eis liegt, mit dem insbesondere die Informationspflichten der Dienstanbieter verstärkt werden sollten. Dieser Gesetzentwurf sollte rasch reaktiviert werden.

Zugleich kündigte der Datenschutzbeauftragte ein härteres Vorgehen gegenüber Internetseiten öffentlicher Stellen in BadenWürttemberg an, die den sog. Like-Button von Facebook in ihre Seiten einbinden. „Ich habe dem Landtag vor kurzem erklärt, dass ich diese Praxis für datenschutzrechtlich nicht zulässig halte, weil dabei sogar Daten von nicht bei Facebook registrierten Nutzern an das soziale Netzwerk übermittelt werden. Wir werden demnächst die Internetauftritte der Behörden überprüfen und ggf. Beanstandungen aussprechen. Vorübergehend bin ich bereit, die sog. ZweiKlick-Lösung zu tolerieren, weil dann Daten nur nach Information des Nutzers und mit dessen aktivem Zutun übermittelt werden“, erläuterte Jörg Klingbeil. Darüber hinaus halte er die Einrichtung von sog. Facebook-Fanpages durch öffentliche Stellen für nicht datenschutzkonform möglich. Auch die Innenministerkonferenz habe auf rechtliche Grauzonen sowie klärungsbedürftige Fragen hingewiesen und öffentliche Stellen aufgrund ihrer Vorbildfunktion zur Zurückhaltung ermahnt. „Diesen Appell kann ich nur unterstreichen", meinte der Landesdatenschutzbeauftragte abschließend. „Es kann nicht angehen, dass öffentliche Stellen quasi Beihilfe dazu leisten, dass die Daten von Nutzern, die sich bei ihnen informieren wollen, zu Geld gemacht werden."

\section{OVG Schleswig-Holstein: Für Facebook gilt kein deutsches Datenschutzrecht}

Mit Beschlüssen vom 22.04.2013 entschied das Schleswig-Holsteinische Oberverwaltungsgericht (OVG) auf Beschwerden des Unabhängigen Landeszentrums für Datenschutz Schleswig-Holstein (ULD) gegen Beschlüsse des Verwaltungsgerichts (VG) Schleswig, dass auf die Datenverarbeitung bei Facebook auch in Bezug auf deutsche Nutzer nicht deutsches, sondern ausschließlich irisches Datenschutzrecht anwendbar sei (Az. 4 MB 10/13, 4 MB 11/13). Da-

1 http://www.bundesrat.de/cln_161/nn_2034972/SharedDocs/ Drucksachen/2011/0101-200/156-11,templateld=raw,property=publicationFile. pdf/156-11.pdf 